\title{
High-Intensity Strength Training Improves Function of Chronically Painful Muscles: Case-Control and RCT Studies
}

\author{
Lars L. Andersen, ${ }^{1}$ Christoffer H. Andersen, ${ }^{1}$ Jørgen H. Skotte, ${ }^{1}$ Charlotte Suetta, \\ Karen Søgaard, ${ }^{3}$ Bengt Saltin, ${ }^{4}$ and Gisela Sjøgaard ${ }^{3}$ \\ ${ }^{1}$ National Research Centre for the Working Environment, Lersø Parkalle 105, 2100 Copenhagen, Denmark \\ ${ }^{2}$ Section of Clinical Physiology and Nuclear Medicine, Department of Diagnostics, Glostrup Hospital, University of Copenhagen, \\ 2600 Glostrup, Denmark \\ ${ }^{3}$ Institute of Sport Science and Clinical Biomechanics, University of Southern Denmark, Campusvej 55, 5230 Odense M, Denmark \\ ${ }^{4}$ Copenhagen Muscle Research Centre, University of Copenhagen, 2100 Copenhagen, Denmark
}

Correspondence should be addressed to Lars L. Andersen; lla@nrcwe.dk

Received 9 November 2013; Revised 2 January 2014; Accepted 5 January 2014; Published 23 February 2014

Academic Editor: David G. Behm

Copyright (C) 2014 Lars L. Andersen et al. This is an open access article distributed under the Creative Commons Attribution License, which permits unrestricted use, distribution, and reproduction in any medium, provided the original work is properly cited.

\begin{abstract}
Aim. This study investigates consequences of chronic neck pain on muscle function and the rehabilitating effects of contrasting interventions. Methods. Women with trapezius myalgia (MYA, $n=42)$ and healthy controls (CON, $n=20)$ participated in a case-control study. Subsequently MYA were randomized to 10 weeks of specific strength training (SST, $n=18$ ), general fitness training (GFT, $n=16$ ), or a reference group without physical training (REF, $n=8$ ). Participants performed tests of 100 consecutive cycles of $2 \mathrm{~s}$ isometric maximal voluntary contractions (MVC) of shoulder elevation followed by $2 \mathrm{~s}$ relaxation at baseline and 10 week follow-up. Results. In the case-control study, peak force, rate of force development, and rate of force relaxation as well as EMG amplitude were lower in MYA than CON throughout all 100 MVC. Muscle fiber capillarization was not significantly different between MYA and CON. In the intervention study, SST improved all force parameters significantly more than the two other groups, to levels comparable to that of CON. This was seen along with muscle fiber hypertrophy and increased capillarization. Conclusion. Women with trapezius myalgia have lower strength capacity during repetitive MVC of the trapezius muscle than healthy controls. High-intensity strength training effectively improves strength capacity during repetitive MVC of the painful trapezius muscle.
\end{abstract}

\section{Introduction}

Neck/shoulder pain is a frequent condition in the working population [1] and pain, tightness, and tenderness of the upper trapezius muscle-trapezius myalgia-are the most common type of chronic neck/shoulder pain [2,3]. In a study among elderly female computer workers with neck/shoulder pain 38\% were clinically diagnosed with trapezius myalgia [3]. In another study among office workers with frequent neck/shoulder pain three out of four experienced tenderness by palpation of the upper trapezius muscle [4]. Trapezius myalgia is associated with pathophysiological changes, such as increased occurrence of ragged red fibers, moth eaten fibers, and cytochrome oxidase negative type I fibers $[5,6]$. Using the microdialysis technique, increased concentration of the algesic substance serotonin during work, stress, and rest has also been shown [7]. Recent studies combining muscle biopsies and microdialysis with gel electrophoresis and mass spectrometry identified several proteins involved in inflammatory processes in myalgic trapezius muscles compared with healthy controls $[8,9]$. Women with trapezius myalgia also have reduced capillarization of type I muscle fibers [10], increased proportion of poorly capillarized type I megafibers [11], impaired regulation of microcirculation locally in the painful trapezius muscle [12-14], and reduced capacity of carbohydrate oxidation [15]. These factors lead to elevated anaerobic metabolism and fatigue during repetitive work $[16,17]$.

In addition, trapezius myalgia negatively impacts muscle functioning. Compared with healthy controls, women with 
trapezius myalgia show decreased maximal voluntary force, rapid force, motor control, endurance, and neck flexibility [18-22]. Trapezius myalgia has also been associated with an inability to properly relax the muscle between repeated contractions [23], which may aggravate development of fatigue. Thus, prevention as well as rehabilitation of neck/shoulder pain has focused on improving different components of physical function such as the ability to relax the muscles using biofeedback training $[24,25]$, muscle coordination training [26], muscle strength using high-intensity resistance training [26-28], endurance using repeated low-intensity contractions [26, 27], fitness training [29], and flexibility using stretching [27]. Among these training modalities strength training appears particularly effective in reducing pain and increasing muscle strength in trapezius myalgia [28]. However, transfer effects of strength training to improve work capacity of the painful trapezius muscle have only been scarcely investigated [26]. This is a potential important aspect, as daily job tasks require repetitive muscle contractions inducing a certain level of fatigue, for example, repetitive arm movements during assembly line work or keyboard typing. Studying the effect of strength training on strengthendurance during repetitive and fatiguing contractions therefore seems pertinent.

A contrasting approach to specific strength training could be to train large muscles distant from the painful site, for example, by general fitness training performed as leg cycling. Previous research in other populations has indicated physiological adaptations in sites distant from the trained muscles [30, 31]. Increased blood flow to the forearms [32] and nonworking limb [33] has also been shown in response to leg exercise. Based on these studies, general fitness training performed as leg cycling may also improve endurance of the painful trapezius muscle.

The aims of the present study were (1) in a casecontrol design to compare muscle function during fatiguing muscle contractions in women with trapezius myalgia and healthy controls and (2) in a randomized controlled trial to investigate the effect of contrasting types of physical rehabilitation in women with trapezius myalgia on muscle function during fatiguing muscle contractions. Additionally, analyses of electromyographic recordings and muscle biopsies were included to investigate neural and muscular adaptations. We hypothesized that specific strength training and general fitness training would be superior to the reference intervention with regard to improved muscle function.

\section{Methods}

2.1. Study Design and Participants. The study design, recruitment of participants, randomization of 48 women, and main results have been described in detail previously $[14,28]$. In the present case-control study, 42 women with trapezius myalgia (MYA, mean \pm SD: $44 \pm 8$ yrs, $165 \pm 6 \mathrm{~cm}, 72 \pm 15 \mathrm{~kg}$, and days with neck pain during previous year $219 \pm 19$ days) and 20 women comparable with regard to job-type, age, weight, and height but without neck muscle complications (CON, mean \pm SD: $45 \pm 9$ yrs, $167 \pm 6 \mathrm{~cm}, 70 \pm 11 \mathrm{~kg}$, and days with neck pain during previous year $5 \pm 6$ days) participated. Exclusion criteria were serious conditions such as previous trauma, life threatening diseases, whiplash injury, cardiovascular diseases, or arthritis in the neck and shoulder. All participants were recruited from workplaces with monotonous and repetitive work tasks, mostly office- and computer-work. All females in MYA were clinically diagnosed with trapezius myalgia, where the main criteria for a positive diagnosis were (1) chronic pain in the neck area, (2) tightness of the upper trapezius muscle, and (3) palpable tenderness of the upper trapezius muscle [28]. In the 10-week intervention study, the 48 women with trapezius myalgia were randomly allocated in balanced design accounting for similar age, BMI, and neck/shoulder trouble to three different 10-week interventions, but 6 dropped out initially in the REF group resulting in 42 women in this study. The random allocation was concealed; that is, the researcher who determined the eligibility of the subjects was unaware which group a subject was to be allocated to. Unfortunately, the timewise successive balanced recruitment resulted in a somewhat smaller REF group, for example, due to withdrawal of 6 participants who initially stated they would volunteer for the study.

All subjects were informed about the purpose and content of the project and gave written informed consent to participate in the study, which conformed to the Declaration of Helsinki and was approved by the Local Ethical Committee (KF 01-138/04). The study was registered in the International Standard Randomised Controlled Trial Number Register (ISRCTN87055459).

\subsection{Measurements and Data Acquisition. Muscle endurance} was measured during consecutive isometric shoulder elevations using a custom-built setup with a steel frame, a chair, and attached force dynamometers (Figure 1). The participant was sitting upright in the height-adjustable chair, and two Bofors dynamometers were placed bilaterally $1 \mathrm{~cm}$ medial to the lateral edge of the acromion with the direction of force being upward [34]. Prior to the endurance test, the participant performed 3 maximal voluntary isometric contractions (MVC) to determine peak force. The participant was then instructed to perform 100 consecutive cycles of 2second MVC followed by 2 -second relaxation. Figure 1 shows the square wave on the computer screen providing visual feedback instructing the participant when to contract and when to relax, with the target value of the square wave set to the peak force determined prior to the endurance test. The participant was instructed to contract as hard and fast as possible when the signal of the square wave went from zero to max and to relax immediately and completely when the signal went from max to zero. The 100 contractions were completed in 6 minutes and 40 seconds.

Electromyography (EMG) signals were recorded synchronously from the upper trapezius muscle with a bipolar surface EMG configuration (Neuroline 720 01-K, Medicotest A/S, Ølstykke, Denmark) and an interelectrode distance of $2 \mathrm{~cm}$ [35]. The electrodes were positioned according to SENIAM guidelines [36]. The skin was abraded prior to applying the electrodes to ensure an impedance of less than $10 \mathrm{k} \Omega$ (typically the impedance was $1-2 \mathrm{k} \Omega$ ). If the impedance was higher than $10 \mathrm{k} \Omega$ the procedure was repeated until 


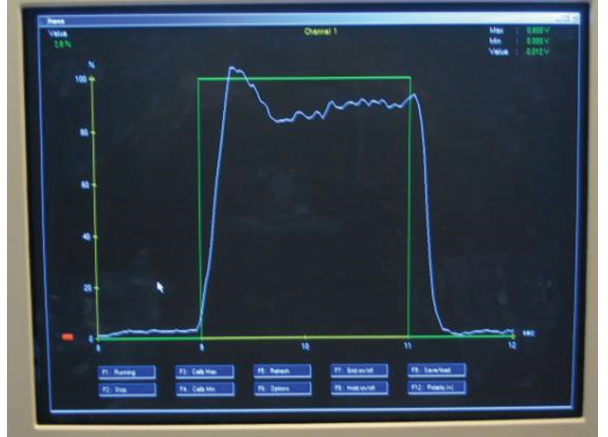

(a)

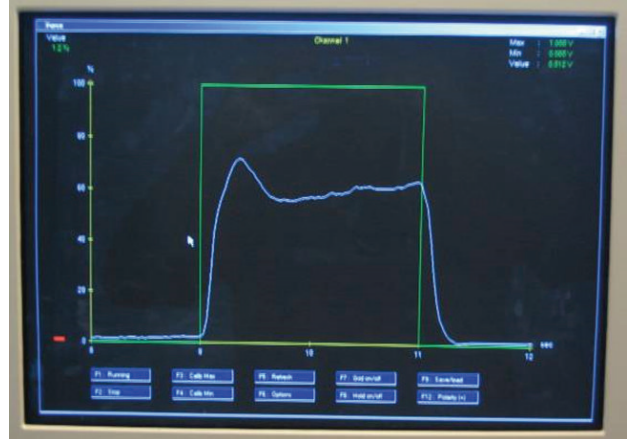

(b)

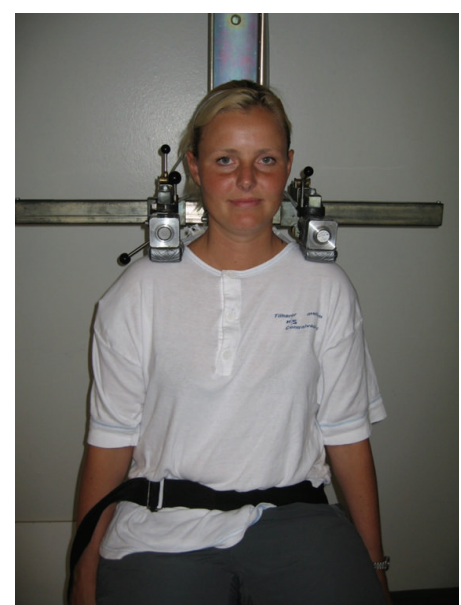

(c)

FIGURE 1: Illustration of experimental setup, the square wave signal (green tracing), and a typical force signal (white tracing) in the beginning (left) and during the end (right) of the 100 consecutive contractions.

impedance was less than $10 \mathrm{k} \Omega$. The EMG electrodes were connected directly to small preamplifiers located near the recording site. The raw analogue EMG signals were led through shielded wires to instrumental differentiation amplifiers, with a bandwidth of $10-400 \mathrm{~Hz}$ and a common mode rejection ratio better than $100 \mathrm{~dB}$. Force and EMG signals were sampled synchronously at $1000 \mathrm{~Hz}$ using a 16-bit A/D converter (DAQ Card-Al-16XE-50, National Instruments, USA) and stored on a laptop for further analysis.

2.3. Data Analysis. Data were analyzed only for the signals from the most painful shoulder. In case of similar pain intensities in both shoulders the dominant side was used for the analyses. During later offline analyses, the force signal was low-pass filtered at $10 \mathrm{~Hz}$, and then three parameters were calculated for each of the 100 MVCs: (1) peak force, determined as the maximal force value within each cycle, (2) rate of force development (RFD) determined as the maximal positive slope over $100 \mathrm{msec}$ of the force signal during the beginning of each MVC, and (3) rate of force relaxation (RFR) determined as the maximal negative slope over $100 \mathrm{msec}$ of the force signal during the end of each MVC. All RFR values were subsequently multiplied by -1 to ease comparison with RFD.

Likewise, the raw EMG signals were filtered using linear EMG envelopes, which consisted of (1) high-pass filtering at
$10 \mathrm{~Hz}$, (2) full-wave rectification, and (3) low-pass filtering at $10 \mathrm{~Hz}$. The filtering algorithms were based on a fourthorder zero phase lag Butterworth filter [37]. From the filtered EMG signal the following parameter was calculated for each of the 100 MVCs: (1) peak EMG, determined as the maximal value of the filtered signal during the top phase of the square signal, that is, when muscle force is peaking, (2) integrated EMG during the first $1 / 2$ second of the top phase of the square signal, that is, during the very beginning of muscle contraction, and (3) resting EMG determined as the average value of the filtered signal during the mid $1 / 2$ second of the bottom phase of the square signal, that is, during relaxation between contractions.

The power spectral density of the EMG signals was calculated as the median power frequency (MPF) in epochs of $1000 \mathrm{~ms}$ in the midphase of each of the 100 contractions. The power density spectra were estimated by Welch's averaged, modified periodogram method in which each epoch was divided into eight Hamming windowed sections with 50\% overlap.

2.4. Muscle Biopsies. Using the needle biopsy technique, muscle biopsies were obtained ultrasound guided from the upper trapezius muscle at the midbelly between the 7 th cervical vertebrae and the acromion. The tissue samples 
were mounted with Tissue-Tek within 2-3 min, frozen in isopentane precooled with liquid nitrogen, and stored in a freezer at $-80^{\circ} \mathrm{C}$ until processed. All biopsy samples were given a unique identification number and blinded. Transverse serial sections $(10 \mu \mathrm{m})$ of the embedded muscle biopsy were cut in a cryostat (Microm, Germany) $\left(22^{\circ} \mathrm{C}\right)$ and mounted on glass slides. Standard ATPase analysis was performed after preincubation at $\mathrm{pH}$ values of $4.37,4.61$, and 10.30 [38]. The biopsy sections were visualized on a computer screen using a Carl Zeiss light microscope (Zeiss Axiolab), a JVC highresolution color digital camera (JVC, TK-C1381EG), and an 8-bit Matrox Meteor Framegrabber (Matrox Electronic Systems, Quebec, Canada). Quantitative analysis of all muscle samples for fiber type percentage, fiber cross-sectional area (CSA), capillaries per fiber (CAF), and capillaries per fiber CSA (CAFA) was performed using a digital image analysis program (TEMA 1.04, Scanbeam, Hadsund, Denmark). All values are reported for types I and II fibers separately. The results on fiber type percentage (case-control study), fiber type area percentage (intervention study), CSA (casecontrol and intervention study), and CAF and CAFA (casecontrol study) have been reported previously [11,39] and are reported here only for comparison with the results from the endurance test. Results from the intervention study for fiber type percentage, CAF, and CAFA have not been reported previously and are reported here as original data.

2.5. Interventions. After the case-control study, the 42 women with trapezius myalgia were randomly allocated to three different 10 -week interventions. The specific strength training group (SST, $n=18$ ) performed five dumbbell exercises specifically for the shoulder and neck muscles (shoulder abduction, shoulder elevation, 1-arm row, reverse flyes, and upright row) for $20 \mathrm{~min}$ three times a week. The high level of activity of the neck and shoulders muscles using these exercises has been documented elsewhere [40]. Three of the five exercises were performed during each session for three sets of each exercise using relative loadings of 8-12 repetitions maximum (RM). The strength training schedule followed principles of progressive overload and periodization as recommended by the ACSM [41]. The general fitness training group (GFT, $n=16$ ) performed leg-bicycling in an upright position with relaxed shoulders on a stationary ergometer at relative loadings of $50 \%$ to $70 \%$ of maximal oxygen uptake for $20 \mathrm{~min}$ three times a week. The loading was estimated based on relative workload $=$ (working heart rate - resting heart rate) $/(\max$ heart rate - resting heart rate), where resting heart rate was set to $70 \mathrm{bpm}$ and max heart rate was estimated as 220 - age [42]. The reference group (REF, $n=8$ ) received information concerning health promotion for one hour per week but were not offered any physical training. Unfortunately, the timewise successive balanced recruitment resulted in a somewhat smaller REF group compared with the two other groups, for example, due to withdrawal of participants who initially stated they would volunteer for the study.

2.6. Statistics. In the case-control study, differences between MYA and CON were tested with two-way ANOVA (Proc
Mixed of SAS). The dependent variables were peak force, RFD, RFR, and peak EMG, respectively. Independent fixed factors included in the model were group (MYA, CON), number (100 contractions), and group by number interaction.

In the intervention study, differences over time between the three groups were tested with repeated measures twoway ANCOVA (Proc Mixed of SAS). The dependent variables were the changes from baseline to follow-up in peak force, RFD, RFR, and peak EMG, respectively. Independent fixed factors included in the model were group (GFT, SST, and REF), number (100 contractions), and group by number interaction. The baseline value of the dependent variable was included as a covariate due to the numerical differences visualized in Figure 2. Participant was entered in the model as a nested random effect using the repeated statement.

The alpha level was set to 0.05 , and results are reported as means \pm SE in the figures and as means and $95 \%$ confidence intervals in the text.

Finally, when statistically significant changes were found we calculated effect sizes as Cohen's $d$ (difference from baseline to follow-up divided by the pooled standard deviation at baseline) [43]. According to Cohen, effect sizes of 0.20 are small, 0.50 moderate and 0.80 large.

\section{Results}

3.1. Case-Control Study. There was a significant group effect for all three force parameters $(P<0.001)$. Post hoc analyses showed that peak force, rate of force development, and rate of force relaxation were lower in MYA compared with CON (Figure 2, left). There was a significant group effect for two of the four EMG parameters. Post hoc analyses showed that peak EMG and rate of EMG rise were lower in MYA compared with CON (Figure 3, left). There was no significant group effect for resting EMG (Figure 3, left) or MPF (Figure 4, left). There was no group by number interaction; that is, the slope over the 100 repetitions did not significantly differ between the two groups, although there was a borderline group by number interaction for MPF $(P=0.05)$.

Capillarization per fiber as well as per fiber area did not significantly differ between MYA and CON, neither for type I nor type II fibers (Table 1).

3.2. Intervention Study. There was a significant group effect for the change from baseline to follow-up for all three force parameters $(P<0.01-0.001)$. Post hoc analyses showed that the strength training group improved significantly more than the two other groups for peak force (SST versus GFT $70 \mathrm{~N}$ [95\% CI 35-105], SST versus REF $50 \mathrm{~N}$ [95\% CI 7-93]), rate of force development (SST versus GFT $346 \mathrm{~N} \cdot \mathrm{s}^{-1}$ [95\% CI 97595], SST versus REF $464 \mathrm{~N} \cdot \mathrm{s}^{-1}$ [95\% CI 156-772]), and rate of force relaxation (SST versus GFT $-378 \mathrm{~N} \cdot \mathrm{s}^{-1}[95 \% \mathrm{CI}-233-$ (-)523], SST versus REF $-323 \mathrm{~N} \cdot \mathrm{s}^{-1}$ [95\% CI -143-(-)503]) (Figure 2, right). The effect sizes for these changes in the SST group were $0.61,0.96$, and 0.90 for peak force, rate of force development, and rate of force relaxation, respectively. Thus, the effects of SST can be considered moderate (peak force) to large (rate of force development and rate of force relaxation). 


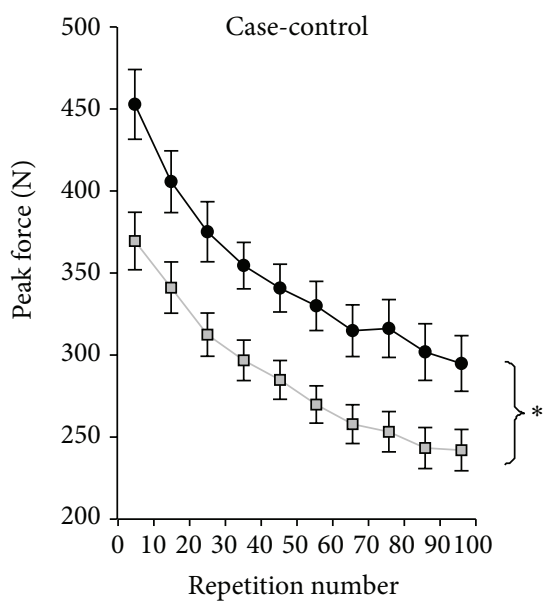

(a)

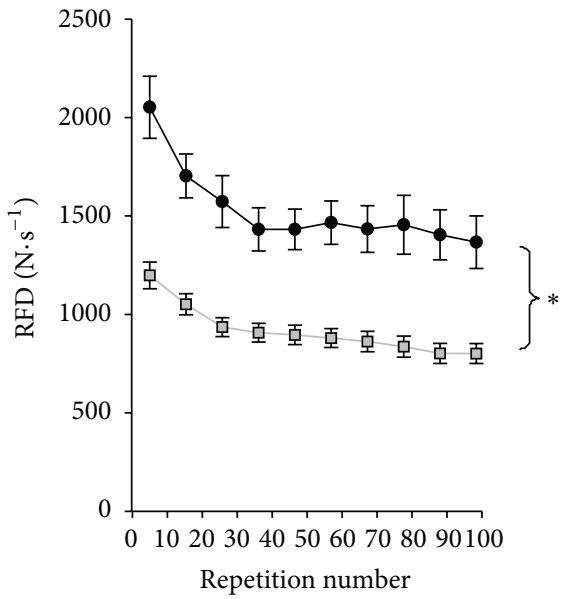

(c)

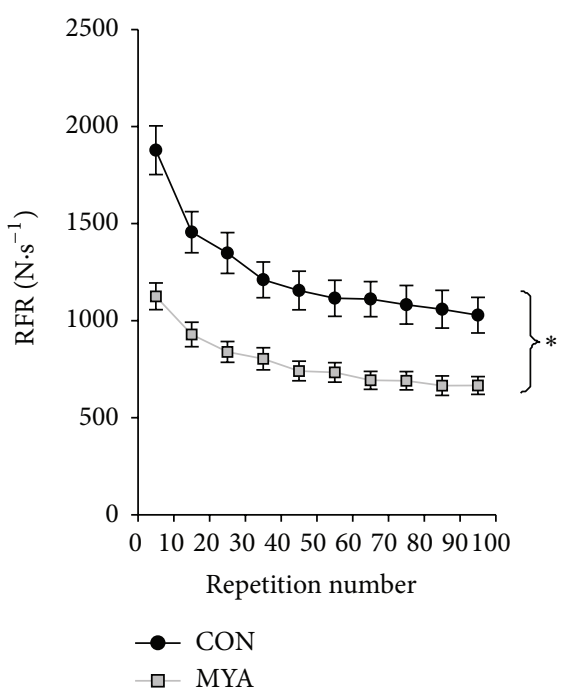

(e)

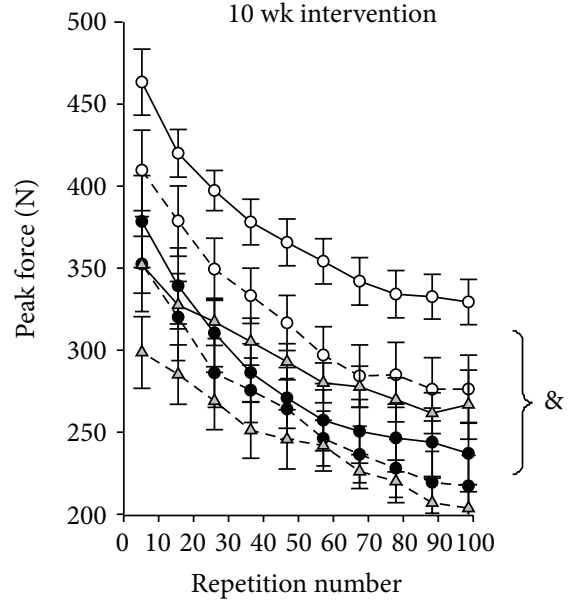

(b)

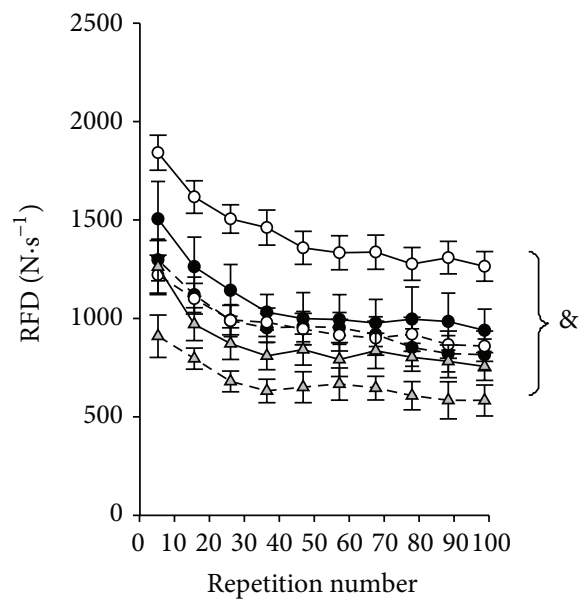

(d)

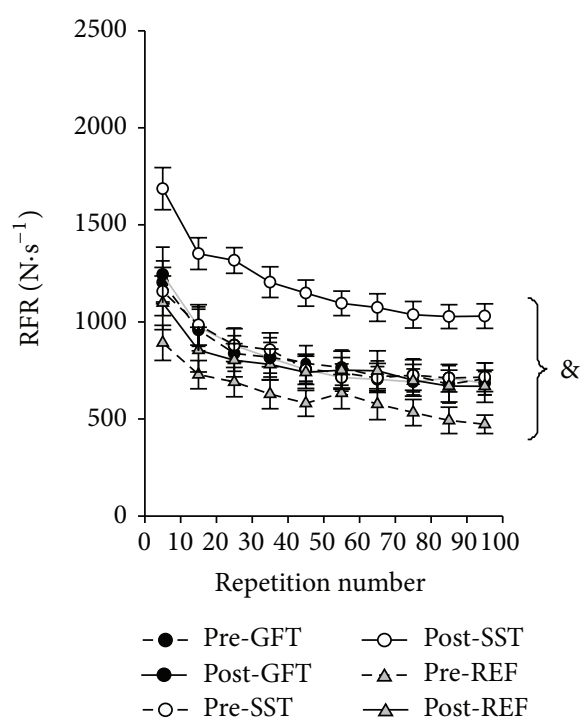

(f)

Figure 2: Case-control (left) and intervention (right) results of peak force (top), rate of force development (RFD) (mid), and rate of force relaxation (RFR) (bottom). ${ }^{*}$ Significant group effect; $P<0.001$ and $P<0.01$. 


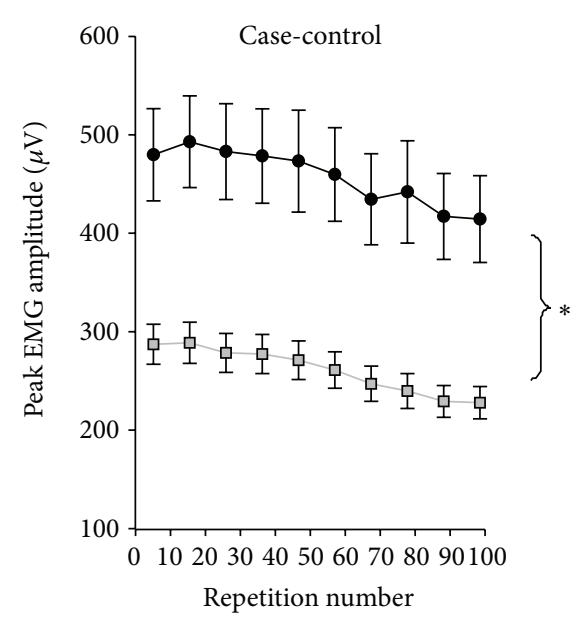

(a)

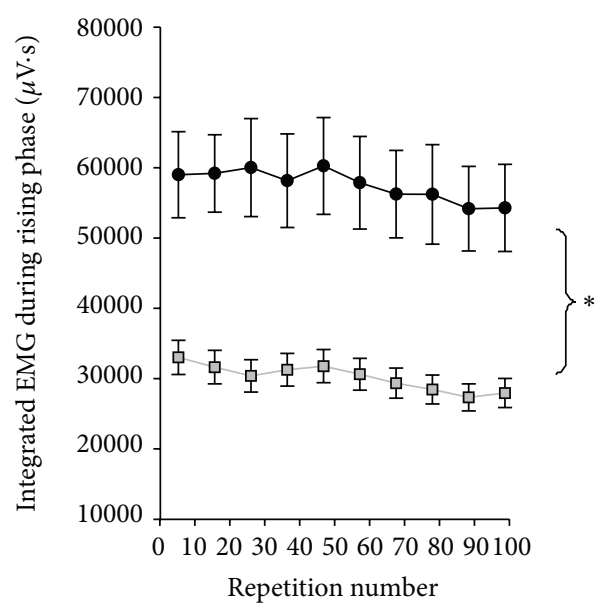

(c)

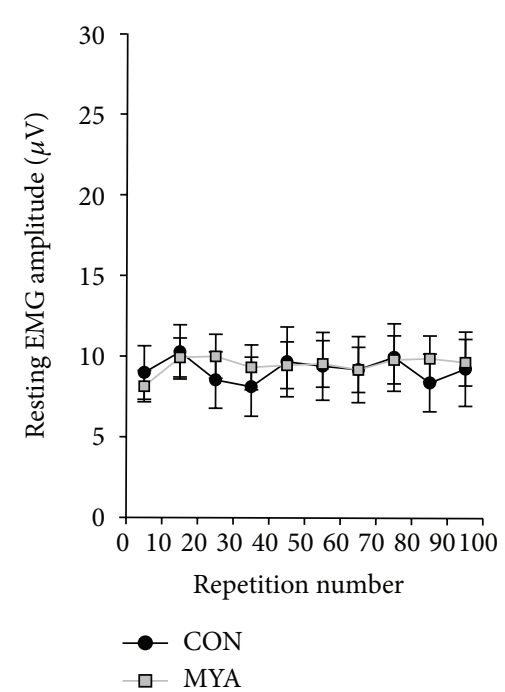

(e)

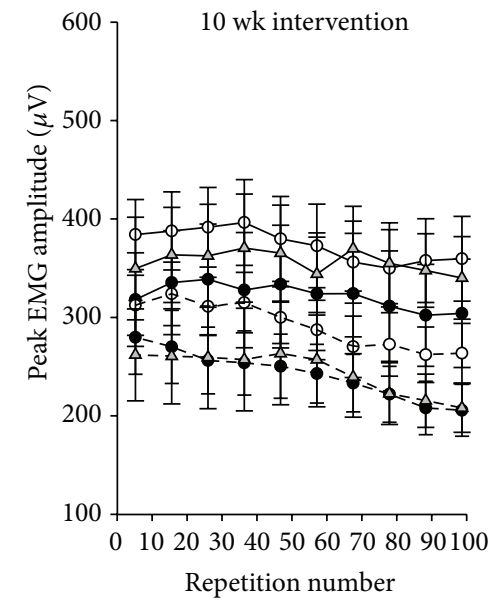

(b)

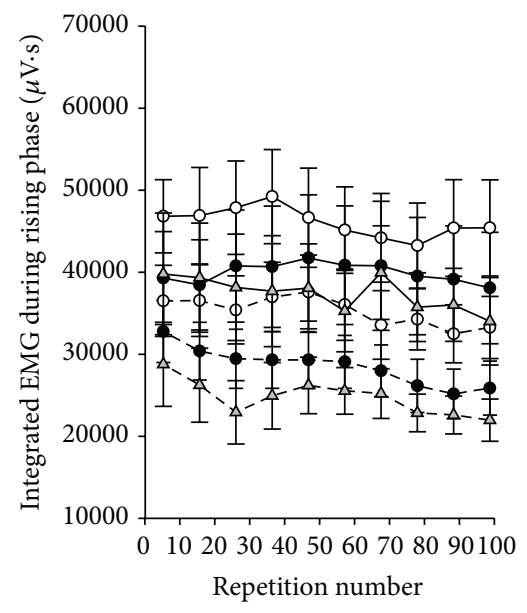

(d)

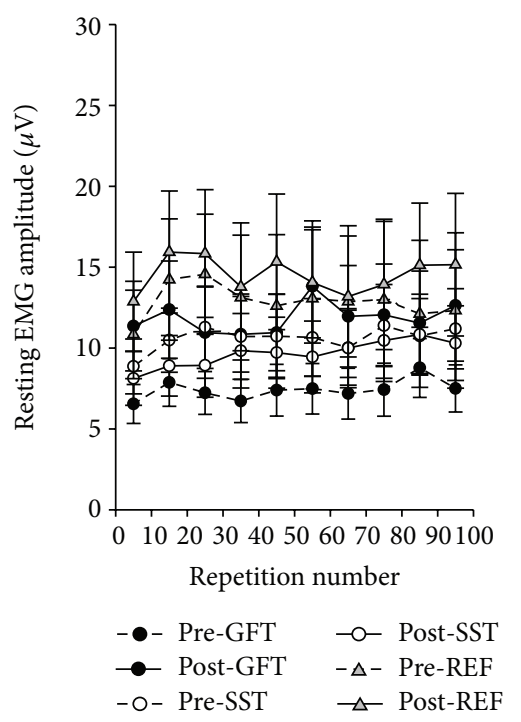

(f)

FIGURE 3: Case-control (left) and intervention (right) results of peak EMG (top), integrated EMG during the rising phase of contraction (mid), and resting EMG (bottom). * Significant group effect; $P<0.001$ and $P<0.01$. 


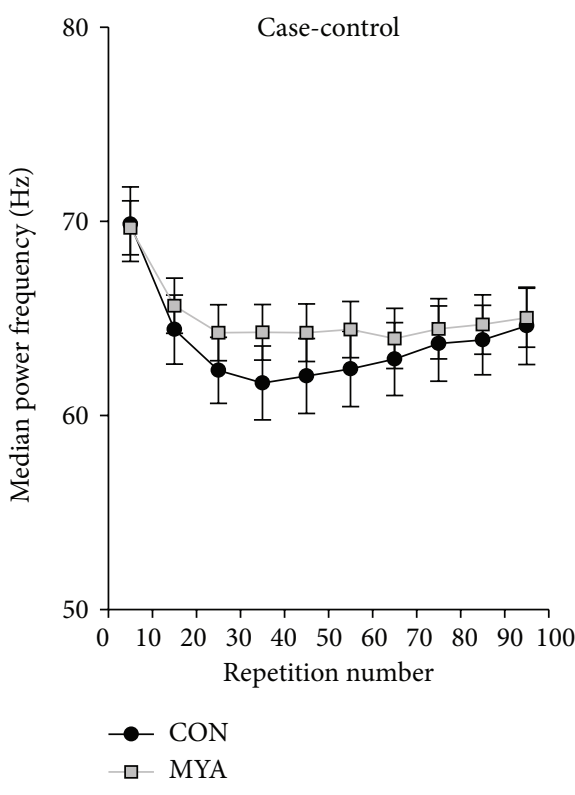

(a)

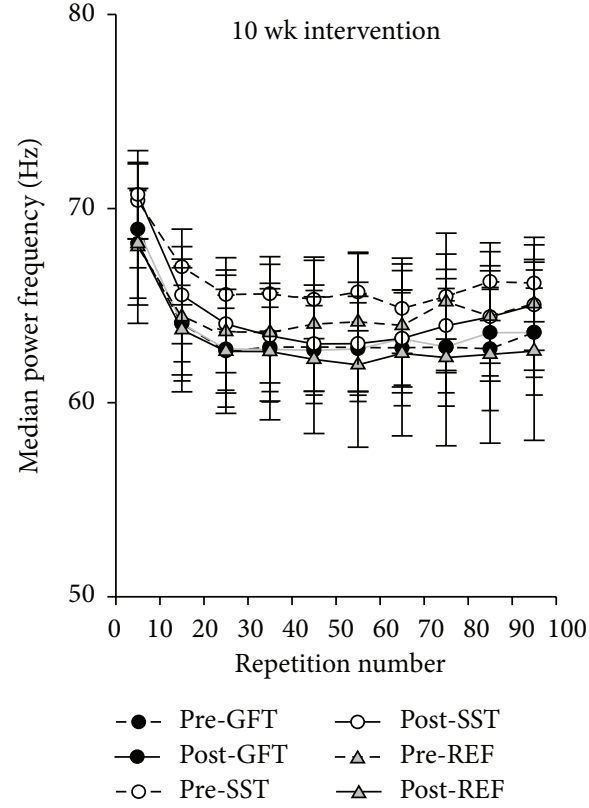

(b)

FIGURE 4: Case-control (left) and intervention (right) results of median power frequency (MPF) of the EMG signal.

TABLE 1: Muscle fiber cross-sectional area, fiber type percentage, capillaries per fiber (CAF), and capillaries per fiber area (CAFA) in healthy controls (CON) and women with trapezius myalgia (MYA) at baseline and before and after 10-week intervention in the specific strength training (SST), general fitness training (GFT), and reference (REF) groups.

\begin{tabular}{|c|c|c|c|c|c|c|c|c|}
\hline & \multicolumn{2}{|c|}{ Area $\left(\mu \mathrm{m}^{2}\right)$} & \multicolumn{2}{|c|}{ Fiber type percentage (\%) } & \multicolumn{2}{|c|}{ Capillaries per fiber (CAF) } & \multicolumn{2}{|c|}{ Capillaries per fiber area (CAFA) } \\
\hline & Type I & Type II & Type I & Type II & Type I & Type II & Type I & Type II \\
\hline $\mathrm{CON}$ & $5057 \pm 1120$ & $4000 \pm 1104$ & $67 \pm 11$ & $33 \pm 11$ & $4.23 \pm 0.74$ & $3.15 \pm 0.72$ & $0.89 \pm 0.15$ & $0.84 \pm 0.17$ \\
\hline MYA & $5193 \pm 1110$ & $3501 \pm 977$ & $69 \pm 11$ & $31 \pm 11$ & $4.12 \pm 0.87$ & $2.79 \pm 0.70$ & $0.83 \pm 0.14$ & $0.86 \pm 0.20$ \\
\hline \multicolumn{9}{|l|}{ REF } \\
\hline Before & $4941 \pm 399$ & $3334 \pm 489$ & $66 \pm 13$ & $34 \pm 13$ & $3.72 \pm 0.49$ & $2.41 \pm 0.33$ & $0.81 \pm 0.14$ & $0.78 \pm 0.14$ \\
\hline After & $5299 \pm 1134$ & $3471 \pm 684$ & $66 \pm 14$ & $34 \pm 14$ & $3.97 \pm 0.53$ & $2.60 \pm 0.61$ & $0.83 \pm 0.18$ & $0.78 \pm 0.16$ \\
\hline \multicolumn{9}{|l|}{ GFT } \\
\hline Before & $5555 \pm 1201$ & $3794 \pm 796$ & $69 \pm 11$ & $31 \pm 11$ & $4.39 \pm 0.80$ & $3.10 \pm 0.51$ & $0.85 \pm 0.19$ & $0.89 \pm 0.25$ \\
\hline After & $5108 \pm 1030$ & $3612 \pm 1070$ & $72 \pm 11$ & $28 \pm 11$ & $4.18 \pm 0.43$ & $2.93 \pm 0.57$ & $0.85 \pm 0.15$ & $0.87 \pm 0.34$ \\
\hline \multicolumn{9}{|l|}{ SST } \\
\hline Before & $5043 \pm 1294$ & $3439 \pm 1331$ & $69 \pm 12$ & $31 \pm 12$ & $4.15 \pm 1.09$ & $2.80 \pm 0.95$ & $0.85 \pm 0.13$ & $0.88 \pm 0.22$ \\
\hline After & $5516 \pm 1188^{\#}$ & $4133 \pm 1145^{\$}$ & $70 \pm 10$ & $31 \pm 10$ & $4.53 \pm 0.82^{\#}$ & $3.31 \pm 0.64^{\$}$ & $0.88 \pm 0.13$ & $0.85 \pm 0.17$ \\
\hline
\end{tabular}

\# Tendency for increase from baseline to follow-up in SST, $P<0.10$.

$\$$ Significant increase from baseline to follow-up in SST, $P<0.05$.

There was no significant group effect for the change from baseline to follow-up for any of the EMG parameters (Figure 3, right, and Figure 4, right). There was no group by number interaction for the change from baseline to follow-up; that is, the change in the slope over the 100 repetitions did not significantly differ between the three intervention groups.

Capillarization per fiber as well as muscle fiber crosssectional area increased significantly in the SST group for type II fibers $(P<0.05)$ and tended to increase for type I fibers $(P<0.10)$. In SST, capillarization per fiber area remained unchanged in both types I and II fibers (Table 1). No significant changes occurred in the two other groups.

3.3. Test-Retest Reliability. Test-retest reliability of the outcome measures was not determined prior to the study. However, we performed reliability analyses after the study using the data from the two groups that did not respond to the intervention, that is, the GFT and REF groups. For these analyses the values for each subject were averaged for the 100 contractions at baseline and follow-up, respectively. 
The intraclass correlation coefficient (ICC) between baseline and 10 -week follow-up was 0.85 for peak force, 0.70 for rate of force development, and 0.85 for rate of force relaxation.

3.4. Post Hoc Power Analysis. Due to the nonsignificant changes in the EMG parameters we performed a post hoc power analysis using the EMG data (averaged for the 100 contractions for each subject) from the two groups that did not respond to the intervention, that is, the GFT and REF groups. At baseline peak EMG was $244 \mathrm{uV}$ (mean value) and the standard deviation of the change from baseline to follow-up was $139 \mathrm{uV}$. Requesting $80 \%$ power to detect $20 \%$ difference from baseline to follow-up with a type I error probability of $5 \%$ would then require 66 subjects in each group. Correspondingly, with only 14 subjects in each group the power to detect $20 \%$ difference is only $20.8 \%$; that is, it is likely that 4 in 5 studies with similar sample size would report null findings.

\section{Discussion}

The main findings of the present study were (1) that women with trapezius myalgia showed reduced force capacity during repetitive maximal contractions of the trapezius muscle compared with healthy controls (case-control study) and (2) that 10 weeks of high-intensity specific strength training led to improved force capacity of the trapezius muscle in women with trapezius myalgia. By contrast, leg cycling did not improve trapezius muscle function. These results along with neural and muscular findings are discussed in the following.

In the case-control study, peak force, rate of force development, and rate of force relaxation as well as peak EMG and integrated EMG during the rising phase of contraction were lower in women with trapezius myalgia compared with healthy controls. We have previously shown-in the same group of subjects-that peak force and rate of force development during a single maximal voluntary contraction are lower in women with trapezius myalgia [21]. In the present study we elaborate on these findings by showing that all these force parameters are lower throughout all 100 contractions. However, the slopes of curves for the peak force values did not differ between the groups. This finding suggests that lower strength-endurance capacity in women with trapezius myalgia is related to the generally lower level of muscle strength - generally requesting a higher relative load during daily life and occupational tasks [14] - rather than faster development of fatigue.

Some of our case-control results contrast previous findings. In contrast to the findings by Larsson and coworkers [10] we did not find reduced capillarization of type I muscle fibers in women with trapezius myalgia compared with healthy controls. This is surprising since we have also in previous studies found a decreased oxygenation in women with trapezius myalgia compared to healthy controls during a more functional and submaximal test using a pegboard task [14]. This may suggest that impaired regulation of microcirculation, rather than reduced capillarization, may contribute to development of pain and fatigue during repetitive work tasks in women with trapezius myalgia.
Also, based on the EMG measurements we found that women with trapezius myalgia and healthy controls had similar ability to relax the muscles, that is, the same level of EMG amplitude, between repeated contractions, which contrast the EMG findings by Elert and coworkers [23]. However, there are methodological differences between the studies which make direct comparison difficult. Elert and coworkers used dynamic contractions and defined the ability to relax the trapezius as the ratio between EMG amplitude of the eccentric (relaxation phase) and concentric phase (contraction phase) of the 100 contractions. As a ratio depends both on the numerator and denominator it can increase simply by decreased maximal EMG during each concentric contraction, which makes the results by Elert and coworkers difficult to interpret.

Different types of normalization of the EMG signal are typically performed to avoid the inherent variance associated with EMG amplitude. By contrast, we did not normalize the EMG amplitude to a maximal voluntary contraction (MVC) as neck/shoulder pain inhibits central drive and thereby the EMG signal during maximal contraction [21, 22, 44]. Thus, normalizing the EMG signal to a maximal contraction would give erroneous normalized values when comparing individuals with and without pain and also when comparing data before and after an intervention that decreases pain. On the other hand, comparing nonnormalized EMG amplitudes between groups is also associated with limitations, such as variance due to electrode placement and thickness of subcutaneous fat. Therefore we carefully placed the EMG electrodes at the same part of the muscle between participants and test sessions by using the seventh cervical vertebrae and the acromion as reference points in accordance with SENIAM guidelines [36]. Further, we have previously reported that there was no difference between MYA and CON in thickness of the subcutaneous layer of fat above the trapezius muscle, and neither did this change significantly during the 10week intervention period [45]. Thus, our results indicate that women with trapezius myalgia have the same ability to relax between muscle contractions.

In spite of similar resting EMG between contractions, the force measurements showed that relaxation from maximal to zero muscle contraction was slower, that is, lower rate of force relaxation, in women with trapezius myalgia than healthy controls. Thus, although women with trapezius myalgia have the ability to fully relax the muscles, it takes longer than in healthy controls. Although speculatively, this may be related to altered $\mathrm{Ca}^{2+}$ kinetics of myalgic muscles. Interestingly, Green and coworkers found a compromised sarcoplasmic reticulum $\mathrm{Ca}^{2+}$-ATPase activity, $\mathrm{Ca}^{2+}$-uptake, and $\mathrm{Ca}^{2+}$-release in a case-series study involving women with myalgia [46]. A slower relaxation of myalgic muscles may have negative implications for high pace work tasks where the time for recovery between contractions is minimal.

The intervention study showed that high-intensity strength training improves both maximal and rapid force capacity as well as the ability to rapidly relax-that is, rate of force relaxation-after each contraction. We have previously reported a marked reduction (79\%) of pain along 
with increased maximal and rapid force capacity of single maximal voluntary contractions in response to the 10 -week high-intensity strength training intervention [28, 39, 47]. The present study elaborates on these findings by showing that muscle function is improved during repetitive maximal muscle contractions. This may have important practical implications as many job tasks require repetitive muscle contractions, such as keyboard typing or repetitive arm movements during assembly line work. Further, previous analyses have shown increased carbohydrate oxidative capacity after specific strength training in women with trapezius myalgia [15], which may also help to explain the ability to sustain a high force development during repetitive contractions. Apart from improved muscle function during repetitive job tasks, the present study also demonstrated significant myofiber hypertrophy and increased capillarization in response to 10 weeks of specific strength training. Thus, these muscular adaptations may partly explain the present findings, although we cannot exclude the existence of neural adaptations mechanisms. Despite the fact that neither of the EMG parameters changed significantly between the intervention groups, the strength training group had the numerically highest EMG amplitude values at 10 -week follow-up. Post hoc power analysis showed that 66 subjects in each group would be required to detect a $20 \%$ difference in peak EMG between groups from baseline to follow-up. Therefore, the present study was underpowered to detect significant changes due to the inherent high variability of the EMG measurements, making significant results more difficult to obtain with a small sample size.

Median power frequency of the EMG signal decreased as expected during fatiguing contractions (Figure 4). This was evident both in healthy controls and women with trapezius myalgia at baseline and follow-up. This validates that the endurance test induces muscle fatigue both among healthy individuals and individuals with pain.

We also included a general fitness training group who performed leg cycling while relaxing the shoulders. Previous research in other populations has shown physiological adaptations in sites distant from the trained muscles [30, 31]. Increased blood flow to the forearms [32] and nonworking limb [33] has also been shown in response to leg exercise. Our hypothesis was that both specific strength training and general fitness training would improve muscle function compared with the reference intervention. However, in the present study leg cycling did not improve muscle fiber capillarization or muscle endurance of the trapezius muscle. Thus, of the three present interventions specific strength training remains the most effective for improving muscle function in women with trapezius myalgia.

In conclusion, the case-control study showed that women with trapezius myalgia have lower strength-endurance capacity during repetitive maximal contractions of the trapezius muscle compared with healthy controls, along with lower muscle activity (EMG amplitude). Moreover, relaxation of force-measured as the rate of force relaxation-occurred more slowly in women with trapezius myalgia, although the ability to fully relax the muscles between contractions was not lower than that in healthy controls. Moreover, the intervention study demonstrated that high-intensity strength training effectively improves strength capacity during repetitive contractions of the painful trapezius muscle attaining functional levels comparable to the healthy control group together with their decrease in pain. This finding was accompanied with muscle fiber hypertrophy and increased capillarization per muscle fiber, which may at least partly explain the functional improvements. By contrast, leg cycling did not improve trapezius muscle function. Collectively, the present findings emphasize the importance of implementing specific resistance exercises in rehabilitation programmes for adults with trapezius myalgia.

\section{Conflict of Interests}

The authors declare that there is no conflict of interests regarding the publication of this paper.

\section{Acknowledgments}

The authors thank all the researchers, assistants, and students of the RAMIN study group. This study was supported by Grants from the Danish Medical Research Council 22-030264 and the Danish Rheumatism Association 233-114902.02.04.

\section{References}

[1] L. L. Andersen, O. S. Mortensen, J. V. Hansen, and H. Burr, "A prospective cohort study on severe pain as a risk factor for long-term sickness absence in blue- and white-collar workers," Occupational and Environmental Medicine, vol. 68, no. 8, pp. 590-592, 2011.

[2] B. Larsson, K. Søgaard, and L. Rosendal, "Work related neckshoulder pain: a review on magnitude, risk factors, biochemical characteristics, clinical picture and preventive interventions," Best Practice and Research: Clinical Rheumatology, vol. 21, no. 3, pp. 447-463, 2007.

[3] B. Juul-Kristensen, R. Kadefors, K. Hansen, P. Byström, L. Sandsjö, and G. Sjøgaard, "Clinical signs and physical function in neck and upper extremities among elderly female computer users: the NEW study," European Journal of Applied Physiology, vol. 96, no. 2, pp. 136-145, 2006.

[4] L. L. Andersen, K. Hansen, O. S. Mortensen, and M. K. Zebis, "Prevalence and anatomical location of muscle tenderness in adults with nonspecific neck/shoulder pain," BMC Musculoskeletal Disorders, vol. 12, article 169, 2011.

[5] B. Visser and J. H. Van Dieën, "Pathophysiology of upper extremity muscle disorders," Journal of Electromyography and Kinesiology, vol. 16, no. 1, pp. 1-16, 2006.

[6] G. M. Hägg, "Human muscle fibre abnormalities related to occupational load," European Journal of Applied Physiology, vol. 83, no. 2-3, pp. 159-165, 2000.

[7] B. Ghafouri, B. K. Larsson, A. Sjörs, P. Leandersson, and B. U. C. Gerdle, "Interstitial concentration of serotonin is increased in myalgic human trapezius muscle during rest, repetitive work and mental stress an in vivo microdialysis study," Scandinavian Journal of Clinical and Laboratory Investigation, vol. 70, no. 7, pp. 478-486, 2010.

[8] P. Olausson, B. Gerdle, N. Ghafouri, B. Larsson, and B. Ghafouri, "Identification of proteins from interstitium of trapezius muscle 
in women with chronic myalgia using microdialysis in combination with proteomics," PLOS ONE, vol. 7, no. 12, Article ID e52560, 2012.

[9] J. Hadrevi, B. Ghafouri, B. Larsson, B. Gerdle, and F. Hellstrom, "Multivariate modeling of proteins related to trapezius myalgia, a comparative study of female cleaners with or without pain," PLoS ONE, vol. 8, no. 9, Article ID e73285, 2013.

[10] B. Larsson, J. Björk, F. Kadi, R. Lindman, and B. Gerdle, "Blood supply and oxidative metabolism in muscle biopsies of female cleaners with and without myalgia," Clinical Journal of Pain, vol. 20, no. 6, pp. 440-446, 2004.

[11] L. L. Andersen, C. Suetta, J. L. Andersen, M. Kjær, and G. Sjøgaard, "Increased proportion of megafibers in chronically painful muscles," Pain, vol. 139, no. 3, pp. 588-593, 2008.

[12] L. L. Andersen, A. K. Blangsted, P. K. Nielsen et al., "Effect of cycling on oxygenation of relaxed neck/shoulder muscles in women with and without chronic pain," European Journal of Applied Physiology, vol. 110, no. 2, pp. 389-394, 2010.

[13] R. Larsson, P. Å. Öberg, and S.-E. Larsson, "Changes of trapezius muscle blood flow and electromyography in chronic neck pain due to trapezius myalgia," Pain, vol. 79 , no. 1, pp. 45-50, 1999.

[14] G. Sjøgaard, L. Rosendal, J. Kristiansen et al., "Muscle oxygenation and glycolysis in females with trapezius myalgia during stress and repetitive work using microdialysis and NIRS," European Journal of Applied Physiology, vol. 108, no. 4, pp. 657669,2010

[15] G. Sjogaard, M. K. Zebis, K. Kiilerich, B. Saltin, and H. Pilegaard, "Exercise training and work task induced metabolic and stress-related mRNA and protein responses in myalgic muscles," BioMed Research International, vol. 2013, Article ID 984523, 12 pages, 2013.

[16] L. Rosendal, A. K. Blangsted, J. Kristiansen et al., "Interstitial muscle lactate, pyruvate and potassium dynamics in the trapezius muscle during repetitive low-force arm movements, measured with microdialysis," Acta Physiologica Scandinavica, vol. 182, no. 4, pp. 379-388, 2004.

[17] L. Rosendal, B. Larsson, J. Kristiansen et al., "Increase in muscle nociceptive substances and anaerobic metabolism in patients with trapezius myalgia: microdialysis in rest and during exercise," Pain, vol. 112, no. 3, pp. 324-334, 2004.

[18] Ó. A. Steingrímsdóttir, S. Knardahl, and N. K. Vøllestad, "Prospective study of the relationship between musculoskeletal and psychological complaints and electromyographic activity during isometric muscular contractions in a working population," Scandinavian Journal of Work, Environment and Health, vol. 30, no. 5, pp. 410-420, 2004.

[19] G. Sjøgaard, K. Søgaard, H. J. Hermens et al., "Neuromuscular assessment in elderly workers with and without work related shoulder/neck trouble: the NEW-study design and physiological findings," European Journal of Applied Physiology, vol. 96, no. 2, pp. 110-121, 2006.

[20] B. R. Jensen, B. Schibye, K. Sogaard, E. B. Simonsen, and G. Sjogaard, "Shoulder muscle load and muscle fatigue among industrial sewing-machine operators," European Journal of Applied Physiology and Occupational Physiology, vol. 67, no. 5, pp. 467-475, 1993.

[21] L. L. Andersen, A. Holtermann, M. B. Jørgensen, and G. Sjøgaard, "Rapid muscle activation and force capacity in conditions of chronic musculoskeletal pain," Clinical Biomechanics, vol. 23, no. 10, pp. 1237-1242, 2008.
[22] L. L. Andersen, P. K. Nielsen, K. Søgaard, C. H. Andersen, J. Skotte, and G. Sjøgaard, "Torque-EMG-velocity relationship in female workers with chronic neck muscle pain," Journal of Biomechanics, vol. 41, no. 9, pp. 2029-2035, 2008.

[23] J. E. Elert, S. B. Rantapaa-Dahlqvist, K. Henriksson-Larsen, R. Lorentzon, and B. U. C. Gerdle, "Muscle performance, electromyography and fibre type composition in fibromyalgia and work-related myalgia," Scandinavian Journal of Rheumatology, vol. 21, no. 1, pp. 28-34, 1992.

[24] L. Dellve, L. Ahlstrom, A. Jonsson et al., "Myofeedback training and intensive muscular strength training to decrease pain and improve work ability among female workers on long-term sick leave with neck pain: a randomized controlled trial," International Archives of Occupational and Environmental Health, vol. 84, no. 3, pp. 335-346, 2011.

[25] A. Holtermann, K. Roeleveld, P. J. Mork et al., "Selective activation of neuromuscular compartments within the human trapezius muscle," Journal of Electromyography and Kinesiology, vol. 19, no. 5, pp. 896-902, 2009.

[26] C. Ahlgren, K. Waling, F. Kadi, M. Djupsjöbacka, L.-E. Thornell, and G. Sundelin, "Effects on physical performance and pain from three dynamic training programs for women with workrelated trapezius myalgia," Journal of Rehabilitation Medicine, vol. 33, no. 4, pp. 162-169, 2001.

[27] J. Ylinen, E. P. Takala, M. Nykänen et al., "Active neck muscle training for treatment of women with chronic neck pain: a randomized controlled study," Manuelle Medizin, vol. 41, no. 6, pp. 491-499, 2003.

[28] L. L. Andersen, M. Kjær, K. Søgaard, L. Hansen, A. I. Kryger, and G. Sjøgaard, "Effect of two contrasting types of physical exercise on chronic neck muscle pain," Arthritis Care and Research, vol. 59, no. 1, pp. 84-91, 2008.

[29] A. K. Blangsted, K. Søgaard, E. A. Hansen, H. Hannerz, and G. Sjøgaard, "One-year randomized controlled trial with different physical-activity programs to reduce musculoskeletal symptoms in the neck and shoulders among office workers," Scandinavian Journal of Work, Environment and Health, vol. 34, no. 1, pp. 55-65, 2008.

[30] P. K. Lunde, I. Sjaastad, H.-M. Schiøtz Thorud, and O. M. Sejersted, "Skeletal muscle disorders in heart failure," Acta Physiologica Scandinavica, vol. 171, no. 3, pp. 277-294, 2001.

[31] N. Yuza, K. Ishida, and M. Miyamura, "Cross transfer effects of muscular endurance during training and detraining," Journal of Sports Medicine and Physical Fitness, vol. 40, no. 2, pp. 110-117, 2000.

[32] D. Silber, D. McLaughlin, and L. Sinoway, "Leg exercise conditioning increases peak forearm blood flow," Journal of Applied Physiology, vol. 71, no. 4, pp. 1568-1573, 1991.

[33] H. Tanaka, S. Shimizu, F. Ohmori et al., "Increase in blood flow and shear stress to nonworking limbs during incremental exercise," Medicine and Science in Sports and Exercise, vol. 38, no. 1, pp. 81-85, 2006.

[34] L. L. Andersen, M. B. Jørgensen, A. K. Blangsted, M. T. Pedersen, E. A. Hansen, and G. Sjøgaard, "A randomized controlled intervention trial to relieve and prevent neck/shoulder pain," Medicine and Science in Sports and Exercise, vol. 40, no. 6, pp. 983-990, 2008.

[35] H. J. Hermens and B. Freriks, The State of the Art on Sensors and Sensor Placement Procedures for Surface Electomyography: A Proposal for Sensor Placement Prodedures. SENIAM Project, Roessingh Research and Development, Enschede, The Netherlands, 1997. 
[36] H. J. Hermens, B. Freriks, C. Disselhorst-Klug, and G. Rau, "Development of recommendations for SEMG sensors and sensor placement procedures," Journal of Electromyography and Kinesiology, vol. 10, no. 5, pp. 361-374, 2000.

[37] D. A. Winter, Biomechanics and Motor Control of Human Movement, John Wiley \& Sons, New York, NY, USA, 2nd edition, 1990.

[38] M. H. Brooke and K. K. Kaiser, "Muscle fiber types: how many and what kind?" Archives of Neurology, vol. 23, no. 4, pp. 369379, 1970 .

[39] L. L. Andersen, J. L. Andersen, C. Suetta, M. Kjær, K. Søgaard, and G. Sjøgaard, "Effect of contrasting physical exercise interventions on rapid force capacity of chronically painful muscles," Journal of Applied Physiology, vol. 107, no. 5, pp. 1413-1419, 2009.

[40] L. L. Andersen, M. Kjær, C. H. Andersen et al., "Muscle activation during selected strength exercises in women with chronic neck muscle pain," Physical Therapy, vol. 88, no. 6, pp. 703-711, 2008.

[41] W. J. Kraemer, K. Adams, E. Cafarelli et al., "Progression models in resistance training for healthy adults," Medicine and Science in Sports and Exercise, vol. 34, no. 2, pp. 364-380, 2002.

[42] P. O. Åstrand and K. Rodahl, Textbook of Work Physiology: Physiological Bases of Exercise, McGraw-Hill, New York, NY, USA, 3rd edition, 1986.

[43] J. Cohen, Statistical Power Analysis for the Behavioral Sciences, Lawrence Erlbaum Associates, 2nd edition, 1988.

[44] A. Ben-Yishay, J. D. Zuckerman, M. Gallagher, and F. Cuomo, "Pain inhibition of shoulder strength in patients with impingement syndrome," Orthopedics, vol. 17, no. 8, pp. 685-688, 1994.

[45] P. K. Nielsen, L. L. Andersen, H. B. Olsen, L. Rosendal, G. Sjøgaard, and K. Søgaard, "Effect of physical training on pain sensitivity and trapezius muscle morphology," Muscle and Nerve, vol. 41, no. 6, pp. 836-844, 2010.

[46] H. J. Green, P. Galvin, D. A. Ranney, H. Tick, and J. Ouyang, "Are abnormalities in sarcoplasmic reticulum calcium cycling properties involved in trapezius myalgia? Case studies on three females," American Journal of Physical Medicine and Rehabilitation, vol. 90, no. 10, pp. 834-843, 2011.

[47] L. L. Andersen, C. H. Andersen, M. K. Zebis, P. K. Nielsen, K. Søgaard, and G. Sjøgaard, "Effect of physical training on function of chronically painful muscles: a randomized controlled trial," Journal of Applied Physiology, vol. 105, no. 6, pp. 1796-1801, 2008. 


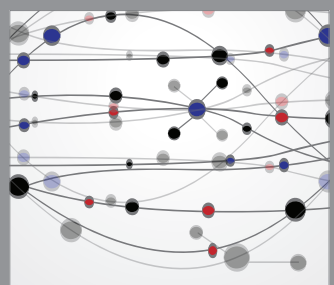

The Scientific World Journal
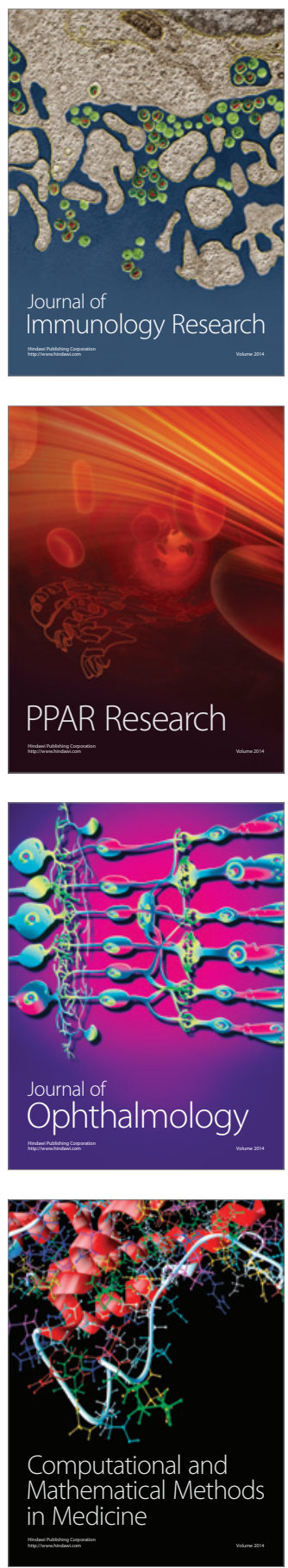

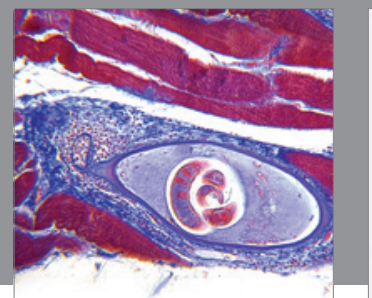

Gastroenterology

Research and Practice
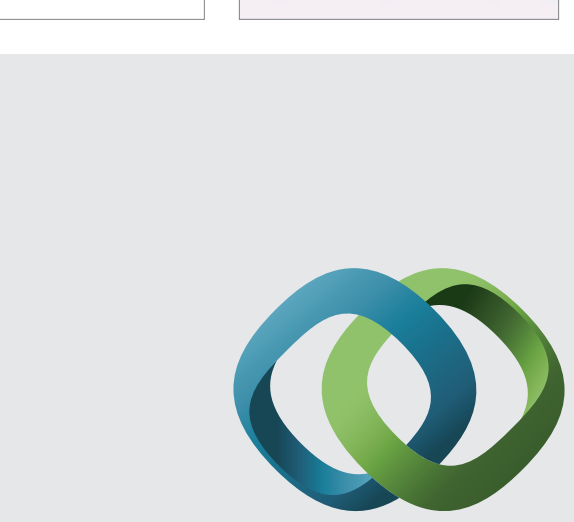

\section{Hindawi}

Submit your manuscripts at

http://www.hindawi.com
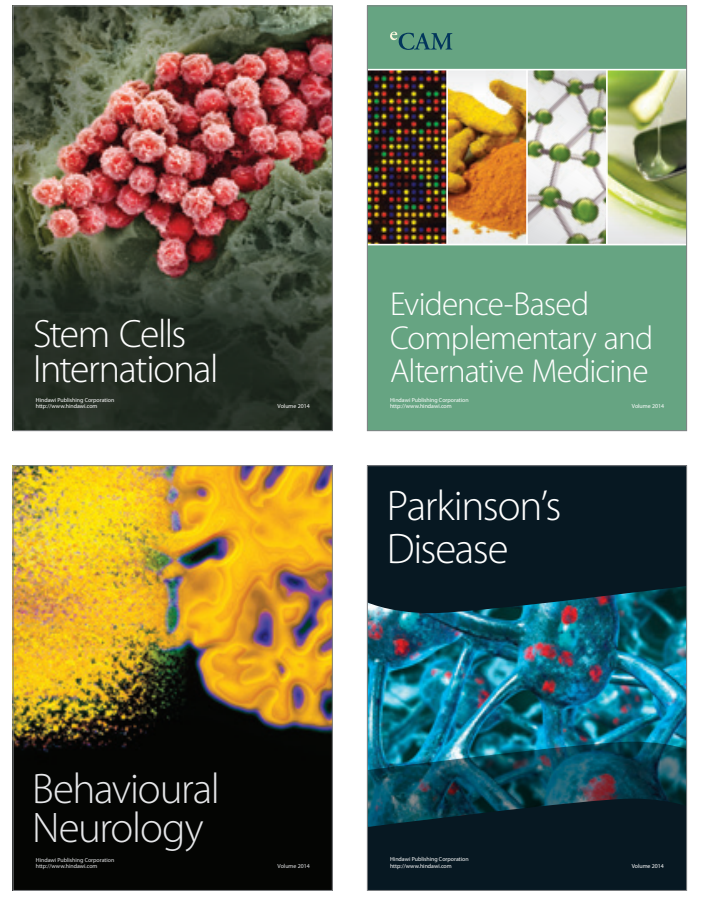
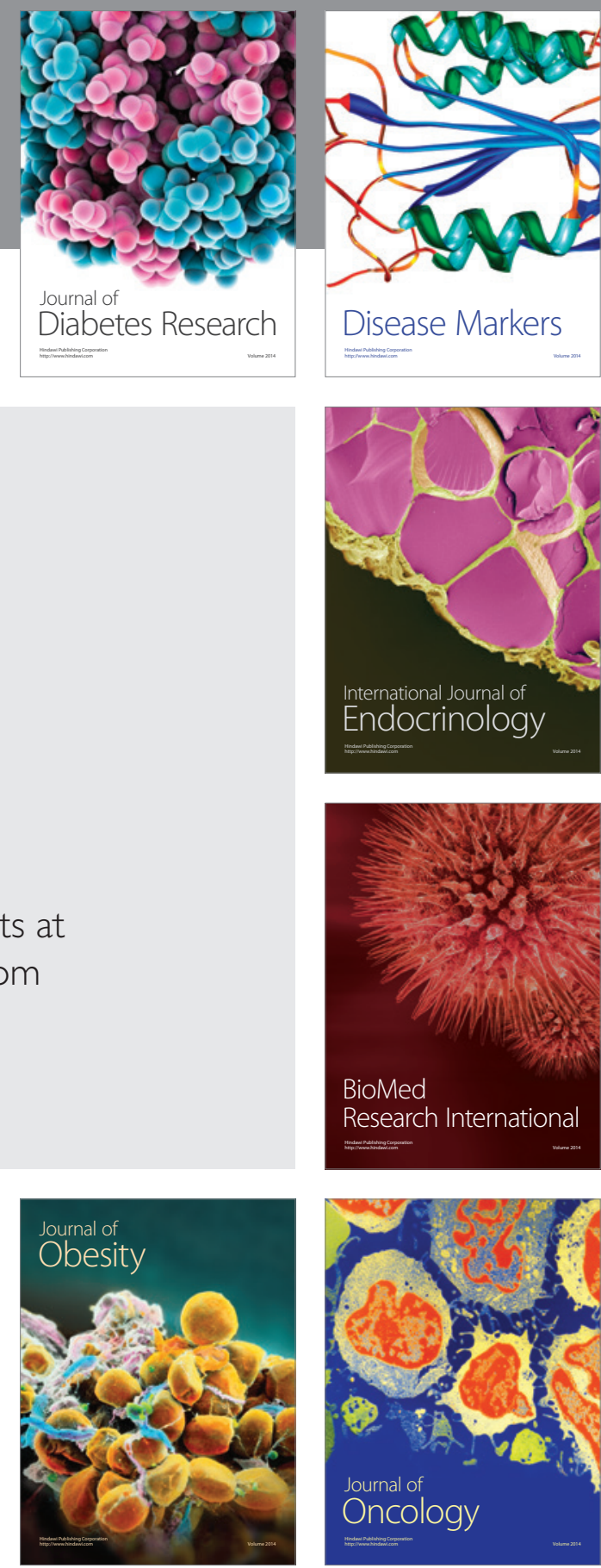

Disease Markers
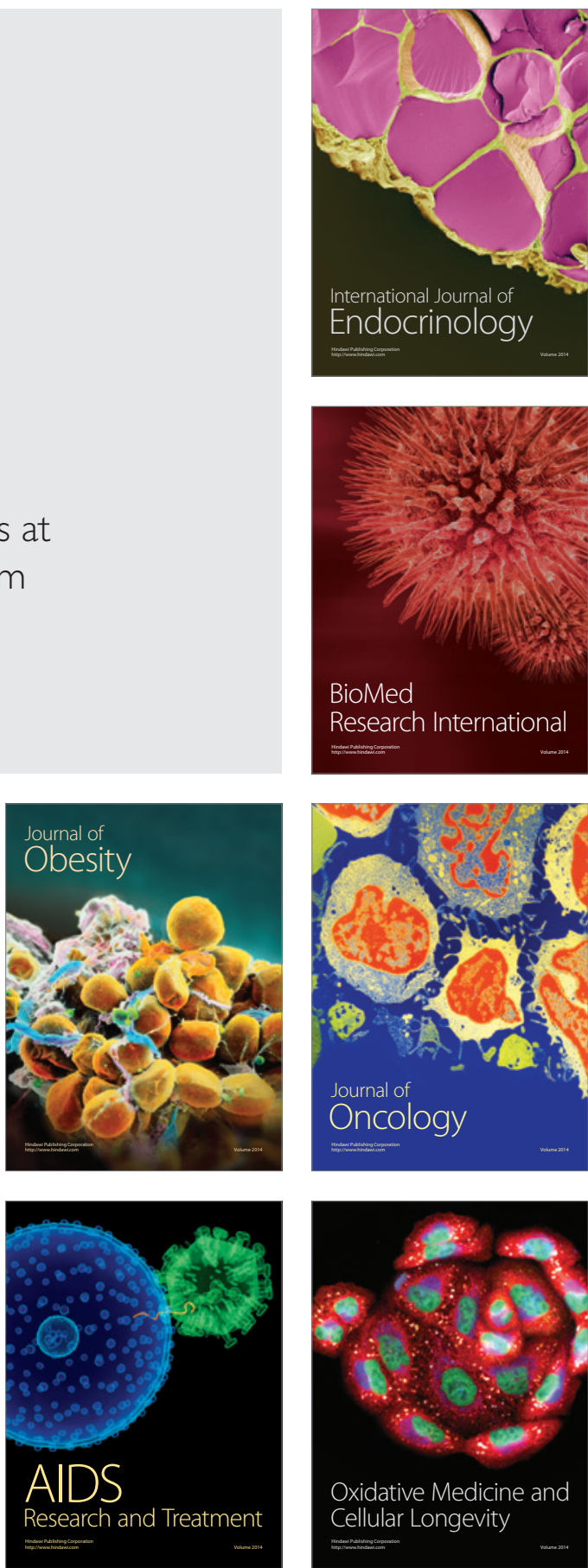